\title{
Late Discovery of a Vein of Galen Aneurysmal Malformation Complicated with Hydrocephalus: A Case Report
}

\section{Diop Abdoulaye*, Faye Mohameth, Cissé Mohamed El Hassimi, Thioub Mbaye, Ba Momar Code, Badiane Seydou Boubakar}

Neurosurgery Department of CHNU Fann, Dakar, Senegal

Email: ^a.diop@univ-zig.sn, fayeneurochir92@gmail.com, elhassimi@yahoo.fr, seybadiane@gmail.com

How to cite this paper: Abdoulaye, D., Mohameth, F., El Hassimi, C.M., Mbaye, T., Code, B.M. and Boubakar, B.S. (2020) Late Discovery of a Vein of Galen Aneurysmal Malformation Complicated with $\mathrm{Hy}$ drocephalus: A Case Report. Open Journal of Modern Neurosurgery, 10, 254-259. https://doi.org/10.4236/ojmn.2020.102027

Received: February 9, 2020

Accepted: March 21, 2020

Published: March 24, 2020

Copyright $\odot 2020$ by author(s) and Scientific Research Publishing Inc. This work is licensed under the Creative Commons Attribution International License (CC BY 4.0)

http://creativecommons.org/licenses/by/4.0/

\begin{abstract}
The aneurysmal malformation of the vein of Galen is a rare condition, corresponding to less than $1 \%$ of intracranial vascular malformations. It is a disease affecting mainly newborns and small children. We are reporting the case of an 11-year-old teenager who presented consciousness disorders associated to generalized convulsive seizures. The cerebral CT scan supplemented by an angioscanner, revealed an important expansive process in the pineal region, confirming the diagnosis of an aneurysmal malformation of the Galen's ampulla with upstream obstructive hydrocephalus. The child benefited from a ventriculo-peritoneal shunt with favorable immediate operative sequels. His death occurred at home two months after surgery over the course of a convulsive seizure with post critical coma.
\end{abstract}

\section{Keywords}

Intracranial Vascular Malformation, Arteriovenous Malformation, Vein of Galen

\section{Introduction}

The vein of Galen aneurysmal malformation is a complex cerebral vascular malformation, leading to a pseudo aneurysmal distention of the Galen's ampulla, which is associated with one or more arteriovenous fistulas. This malformation results from the lack of differentiation of a primitive median embryonic vein into a mature arteriovenous system, draining the primitive choroid plexus, a phenomenon that occurs usually between the seventh and twelfth week of amenorrhea [1]. It is a rare malformation and a very particular one because of its semio- 
logical aspects, therapeutic difficulties and prognostic severity. It accounts though for less than $1 \%$ of intracranial arteriovenous malformations [2]. It may occur in the neonatal period, usually in the first days of life, through congestive heart failure, or later in childhood [3]. The reported case concerns an aneurysmal malformation of Galen's ampulla. It has been lately discovered and complicated with an obstructive hydrocephalus in an 11-year-old teenager. It was treated in the neurosurgery department of the Fann University Hospital Center and its evolution was fatal.

\section{Clinical Case}

This is about an 11-year-old teenager with a history of generalized convulsive seizures evolving since his infancy, who had not been treated before. He was referred to the neurosurgical emergency unit by a peripheral health structure for a disturbance of consciousness in a context of irritability, diffuse headache and vomiting. Neurological examination revealed an altered consciousness with a Glasgow Coma Scale rated to 9 (E2M5V2), bilateral loss of visual acuity and meningeal syndrome. The dilated fundus examination showed bilateral papillary edema. The examination of the cephalic area allowed to find a thrilling medio-parietal swelling and a turgidity of the scalp veins. Cardiovascular examination revealed a regular tachycardia without murmur. The rest of the examination was within normal limits.

The cerebral CT scan showed a mass in the pineal region with peripheral calcifications, which was intensely enhanced after of the contrast material injection. It happens to be a voluminous aneurysmal expansion of the great vein of Galen, responsible for an upstream active hydrocephalus (Figure 1). The cerebral CT angiography showed that the aneurysm was supplied by a double arterial afference. The first one goes from the left posterior cerebral artery through a $10 \mathrm{~mm}$ left lateral collar, and the other one goes from a distal branch of the right posterior
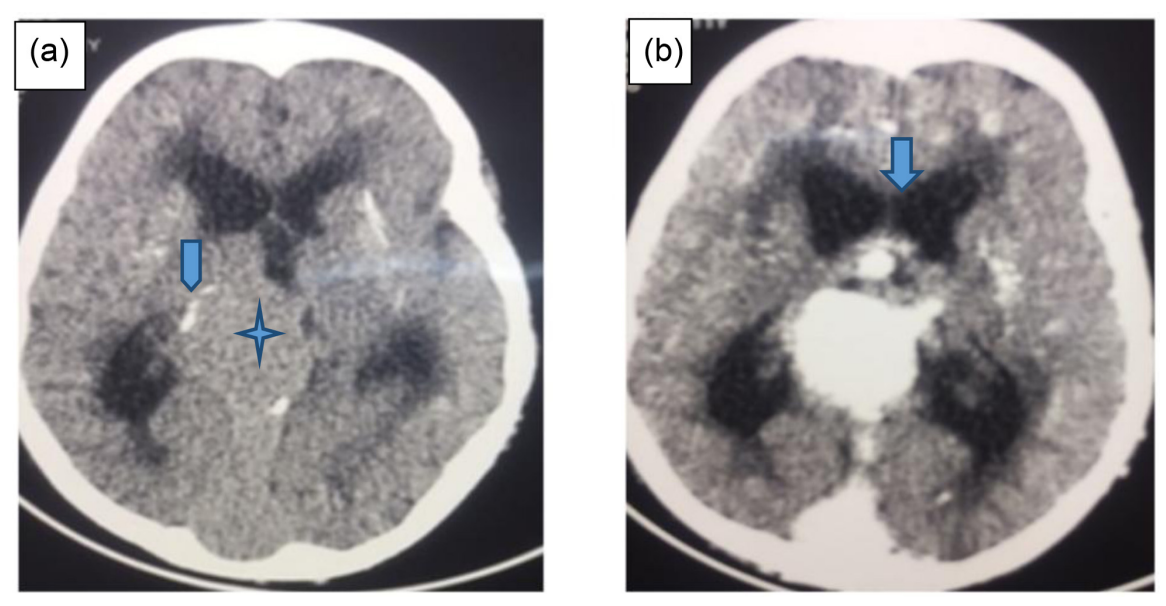

Figure 1. Brain CT scan in axial section, before contrast material injection (a) and after contrast material injection (b) showing the complex of the aneurysm of Galen's vein ( $t$ ) with peripheral calcifications $(\mathbb{Z})$ and an upstream hydrocephalus $(\mathbb{V})$. 
cerebral artery feeding directly the Galen's vein through a $7.5 \mathrm{~mm}$ anterior collar (Figure 2). The echocardiography performed returned normal.

Due to the poor clinical condition of the patient at the entrance as well as the inaccessibility of an endovascular treatment, a ventriculo-peritoneal shunt was the only therapeutic alternative in our context and was performed on the patient.

The short-term postoperative course was favourable with a recovery of consciousness and an improvement of the intracranial hypertension. The follow-up cerebral CT scan performed two days after surgery was satisfying (Figure 3). The child was discharged from the hospital with antiepileptic treatment on the fifth day after surgery. He died at home two months after a convulsive seizure with post critical coma.
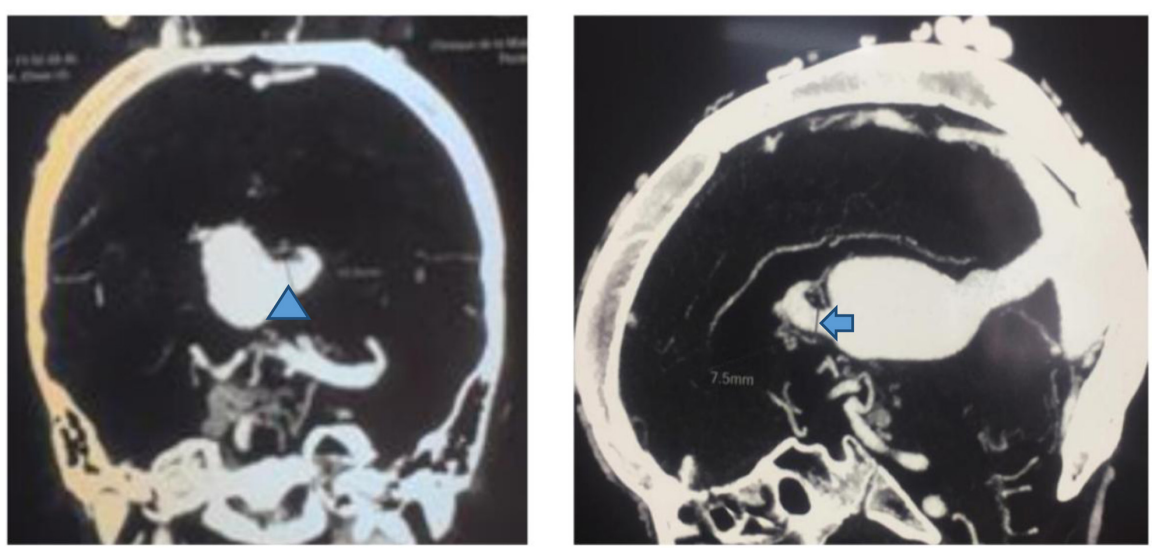

Figure 2. Cerebral CT angiography showing the aneurysm double blood afference from the left posterior cerebral artery through a side left collar $(10 \mathrm{~mm})(\Delta)$ and from a distal branch of the right posterior cerebral supplying directly the Galen vein by a anterior collar $7.5 \mathrm{~mm}$ ( ) draining into the vein of Galen which is expanded with obstructive hydrocephalus.

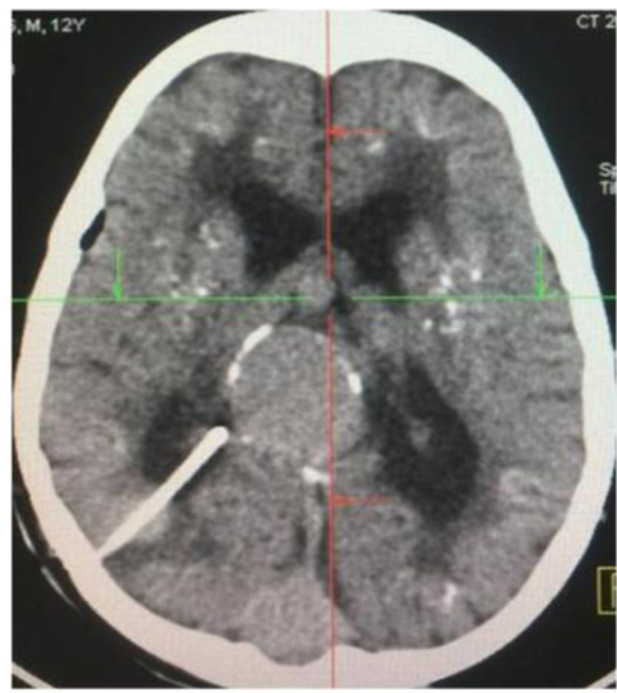

Figure 3. Cerebral CT Scan without contrast material injection showing the intraventricular catheter (J-2 postoperative). 


\section{Discussion}

Aneurysmal malformation of the Galen's ampulla is very rare, corresponding to less than $1 \%$ of all cerebral vascular malformations [2]. The great majority of cases of aneurysmal malformation of the Galen's vein are diagnosed between 1 and 12 months [3]. Concerning our patient, the diagnosis delay can be explained by the low socioeconomic level of the family and their remoteness from health care facilities. Abe T [4] and Xu D.S [5] reported cases of late diagnosis at 19 and 44 years of age respectively. In older children, clinical signs are usually convulsive seizures, neurological deficits and psychomotor delays [3] [6]. In our observation, intracranial hypertension was the main finding circumstance.

The mechanism of the hydrocephalus related to the malformation was not clarified in our case. It was due either to direct compression of the Sylvius' aqueduct by the dilated vein, or to an increase of intracranial pressure leading to a decrease in cerebrospinal fluid resorption [7]. Hydrocephalus may appear shortly after birth or in early childhood, often representing the first telltale sign of the malformation. It may then be responsible for irritability, consciousness disorders or convulsions [7].

Endovascular treatment is currently the best treatment option with a success rate and overall survival of about $80 \%$. Ventriculo-peritoneal shunting may be an alternative treatment for Galen's ampulla malformations complicated with hydrocephalus [8] [9]. However, ventriculo-peritoneal shunt as first-line treatment has many short- and long-term disadvantages, including persistent epilepsy, intraventricular hemorrhage, and subdural hematoma. These complications can lead to rapid neurological deterioration and eventually death [7]. Yasargil et al., reported two cases of postoperative deaths related to intraventricular hematomas in patients presenting with hydrocephalus due to Galen's vein aneurysm [10]. In the Dan Meila el al. [7] series, out of seven patients operated on for hydrocephalus complicating a Galen's ampulla malformation, six ventriculo-peritoneal shunting showed severe postoperative complications. Therefore, ventriculo-peritoneal shunting should be limited to cases of symptomatic hydrocephalus in which the patient is a poor candidate for embolization or in cases where endovascular treatment has already been optimized [11]. Our patient was received in the hospital with an acute hydrocephalus and a ventriculo-peritoneal shunt seemed to be the best therapeutic indication in our context. He did not have any follow-up imaging at the time of his neurological aggravation at home and therefore his death cannot be formally attributed to a valve complication.

\section{Conclusion}

Galen' vein aneurysmal malformation is a rare disease whose prognosis is often unfavorable in our context of exercise. For older children, the discovery of a clinically well supported malformation of the great Galen's vein should lead to the only fully effective treatment, which is endovascular occlusion. Hydrocephalus complicating a Galen's ampulla malformation may lead to discussion of a 
ventricular bypass valve or ventriculo cisternostomy. A better understanding of the angioarchitecture, a better timing of treatment methods and associated complications can increase global survival and quality of life for the carriers of these malformations.

\section{Acknowledgements}

We thank everyone who participated in the development of this work.

\section{Conflicts of Interest}

On behalf of all author, the corresponding author states that there is no conflict of interest.

\section{References}

[1] Raybaud, C.A., Strother, C.M. and Hald, J.K. (1989) Aneurysms of the Vein of Galen: Embryonic Considerations and Anatomical Features Relating to the Pathogenesis of the Malformation. Neuroradiology, 31, 109-128.

https://doi.org/10.1007/BF00698838

[2] Kurihara, N., Tokieda, K., Ikeda, K., Mori, K., Hokuto, I., Nishimura, O., et al. (2001) Prenatal MR Findings in a Case of Aneurysm of the Vein of Galen. Pediatric Radiology, 31, 160-162. https://doi.org/10.1007/s002470000389

[3] Lasjaunias, P., Terbrugge, K., Piske, R., Lopez Ibor, L. and Manelfe, C. (1987) Dilatation de la veine de Galien. Formes anatomo-cliniques et traitement endovasculaire à propos de 14 cas explorés et/ou traités entre 1983 et 1986. Neurochirurgie, 33, 315-333.

[4] Abe, T., Matsumoto, K., Kiyota, K. and Tanaka, H. (1996) Vein of Galen Aneurysmal Malformation in an Adult: A Case Report. Surgical Neurology, 45, 39-43. https://doi.org/10.1016/0090-3019(95)00340-1

[5] Xu, D.S., Usman, A.A., Hurley, M.C., Eddleman, C.S. and Bendok, B.R. (2010) Adult Presentation of a Familial-Associated Vein of Galen Aneurysmal Malformation: Case Report. Neurosurgery, 67, E1845-E1851. https://doi.org/10.1227/NEU.0b013e3181fa00d8

[6] Khullar, D., Andeejani, A.M.I. and Bulsara, K.R. (2010) Evolution of Treatment Options for Vein of Galen Malformations. Journal of Neurosurgery Pediatrics, 6, 444-451. https://doi.org/10.3171/2010.8.PEDS10231

[7] Meila, D., Grieb, D., Melber, K., et al. (2016) Hydrocephalus in Vein of Galen Malformation: Etiologies and Therapeutic Management Implications. Acta Neurochirurgica (Wien), 158, 1279-1284. https://doi.org/10.1007/s00701-016-2836-y

[8] Jones, B.V., Ball, W.S., Tomsick, T.A., Millard, J. and Crone, K.R. (2002) Vein of Galen Aneurysmal Malformation: Diagnosis and Treatment of 13 Children with Extended Clinical Follow-Up. American Journal of Neuroradiology, 23, 1717-1724.

[9] Johnston, I.H., Whittle, I.R., Besser, M. and Morgan, M.K. (1987) Vein of Galen Malformation: Diagnosis and Management. Neurosurgery, 20, 747-758. https://doi.org/10.1227/00006123-198705000-00013

[10] Yasargil, M.G., Antic, J., Laciga, R., Jain, K.K. and Boone, S.C. (1976) Arteriovenous Malformations of Vein of Galen: Microsurgical Treatment. Surgical Neurology, No. 3, 195-200. 
[11] Champeaux, C., Botella, C., Lefevre, E., et al. (2016) Obstructive Hydrocephalus Caused by an Unruptured Arteriovenous Malformation Successfully Treated by Endoscopic Third Ventriculostomy after Shunt Dysfunction. Turkish Neurosurgery, 28, 500-504. https://doi.org/10.5137/1019-5149.JTN.19435-16.2 